\title{
Care for Older People with Dementia During COVID-19 Pandemic
}

\author{
Demelza Emmerton ${ }^{1} \cdot$ Ahmed H. Abdelhafiz ${ }^{1}$ \\ Accepted: 16 December 2020 / Published online: 27 January 2021 \\ (C) The Author(s), under exclusive licence to Springer Nature Switzerland AG part of Springer Nature 2021
}

\begin{abstract}
Older people living with dementia, who are likely frail with multiple comorbidities, appear particularly vulnerable to COVID-19. Care for older people with comorbid dementia and COVID-19 is a challenge to health care professionals due to their complex needs. COVID-19 is a respiratory disease which typically presents with respiratory symptoms; however, in older people with dementia, it may present atypically with delirium. Delirium may precede respiratory symptoms, and in some cases, it may be the only symptom, leading to a delay in the diagnosis. Therefore, screening for delirium should be part of the routine clinical practice for older people with dementia and suspected COVID-19 infection. Due to the complexity of care required for older people with dementia affected by COVID-19, a holistic and individualised approach that includes acute, transitional and long-term care is required. Advanced decision-making, for example, ceiling of care and resuscitation decisions, should be made early on admission to hospital. Screening for frailty with clinical frailty scale may help to aid decision-making. Palliative care and relief of suffering should be considered from the outset. Early and regular involvement of patients and their families in care plans and periodic updates regarding any changes in the clinical condition are good clinical practice. The introduction of telehealth programmes that are suitable for older people with poor cognitive function and also cover diverse cultural backgrounds are urgently required for the future support of this vulnerable group of patients.
\end{abstract}

Keywords COVID-19 $\cdot$ Older people $\cdot$ Risk factors $\cdot$ Dementia $\cdot$ Management

\section{Introduction}

Older people are particularly vulnerable to natural disasters and crisis, and this has been especially true during the COVID-19 pandemic. The mortality of COVID-19 patients was found to be proportionally higher with increasing age especially in those with pre-existing comorbidities [1]. An overall fatality rate of $2.3 \%$ has been reported across all age groups, rising to $8.0 \%$ in patients aged $70-79$ years and significantly higher (14.8\%) in those $\geq 80$ years old [1]. The COVID-19 virus spreads rapidly by human-to-human transmission, primarily through respiratory droplets, and typically presents with respiratory, gastrointestinal or general symptoms [2]. However, in older people, COVID-19 may present atypically with acute confusion or delirium, especially in

This article is part of the Topical Collection on Covid-19

Ahmed H. Abdelhafiz

ahmedhafiz@hotmail.com

1 Department of Geriatric Medicine, Rotherham General Hospital, Moorgate Road, Rotherham S60 2UD, UK patients with pre-existing cognitive dysfunction or dementia. This has been previously shown in older people affected by the SARS epidemic in 2003 who presented not only with respiratory symptoms but also with delirium [3]. This manuscript reviews the atypical neurological presentation of COVID-19 in dementia patients and methods of prevention, diagnosis and management.

\section{Dementia}

Dementia is a syndrome of progressive deterioration in memory, thinking, behaviour and the ability to perform activities of daily living beyond what might be expected within normal ageing. The prevalence and incidence of dementia is agerelated and is associated with increased prevalence of disability, poor quality of life, institutionalisation and mortality. Currently, the prevalence of dementia is about 50 million people worldwide and the incidence is around 10 million new cases per year. There is often a lack of awareness and understanding of dementia which may result in delayed diagnosis and barriers to appropriate care. Dementia has a physical, psychological, social and economic impact, not only on 
people with the disease but also on their carers, families and the society at large [4]. It can be physically, emotionally and financially overwhelming for families to care for people with dementia leading to significant stress, and therefore, support is almost always required from health and social services.

\section{COVID-19 and Dementia—the Link}

Ageing, frailty and comorbidity are common risk factors for the development of dementia and have been synergistically associated with COVID-19 infection and adverse outcomes [5]. Analysis of 17,425,445 NHS-registered adults in the UK has shown a mortality of 5683 due to COVID-19 which was proportionally related to age. Compared to the age group of 50-58 years old as a reference point, the adjusted mortality risk was only 0.07 (95\% CI 0.05 to 0.1 ) in people between 18 and $<40$ years of age, while in older people $\geq 80$ years, the risk was 12.64-fold (95\% CI 11.19 to 14.28) [6]. The number of comorbidities also proportionally increases the risk of COVID-related adverse outcomes [7]. Comorbidity associated with increased age leads to the emergence of complex health states that tend to occur later in life and commonly called geriatric syndromes that include dementia and frailty. Frailty, as a result of ageing and comorbidity, is a risk factor for dementia. It has been shown that frail older people, mean (SD) age 82 (4.6) years, are 8 times more likely to have cognitive impairment (odds ratio (OR) 7.8, 95\% confidence interval (CI) 4.0 to 15.0$)$ or dementia $(8.0,4.0$ to 15.9$)$. They were also about 6 times more likely to have vascular dementia (5.6, 1.2 to 25.8 ) and over 4 times more likely to have Alzheimer's disease $(4.5,2.1$ to 9.6), and presence of dementia accelerates the rate of the progression of frailty $[8,9]$. Recently, in a multicentre European cohort study, frailty assessed by the clinical frailty score (CFS) proportionately predicted COVID-19 mortality. Compared with CFS 1-2, the adjusted hazard ratios for time from hospital admission to death were 1.55 (95\% CI 1.00 to 2.41) for CFS 3-4, 1.83 (1.15 to 2.91) for CFS 5-6 and 2.39 (1.50 to 3.81) for CFS 7-9, and adjusted odds ratios for day 7 mortality were 1.22 (95\% CI 0.63 to 2.38) for CFS 3-4, 1.62 (0.81 to 3.26) for CFS 5-6 and 3.12 (1.56 to 6.24) for CFS 7-9 [10]. Therefore, it appears that there is link between dementia and COVID-19 infection mediated by direct risk factors of ageing, comorbidity and frailty.

\section{COVID-19 and Dementia—the Risk}

Normally, people living with dementia are the most vulnerable and dependent group in the society. They are relying on the care delivered by family members or professional carers for their day to day survival. During the COVID-19 pandemic, their vulnerability is likely to be exaggerated due to the direct impact of the disease on them and the indirect effects of the pandemic on the social support and the health care system. Older people with dementia are likely to have little information or understanding of the COVID-19 pandemic. Health care safeguarding procedures such as social distancing, hand washing, wearing masks or reporting early symptoms of the infection may be difficult tasks to comprehend in this population exposing them to a higher risk of infection. With increasing social isolation, people with dementia and living alone may have less carer input which may aggravate their isolation, increasing their loneliness and risk of depression and anxiety. More ever, patients with severe dementia and behavioural problems such as agitation or wandering will undermine every effort to maintain isolation and can be a risk for other individuals especially their carers. The outcome of COVID-19 is likely to be worse when dementia is present. Older people with dementia are likely to have high prevalence of comorbid conditions such as cardiovascular disease, diabetes and increased risk of pneumonia compared to individuals without dementia [11]. These comorbidities have been associated with adverse outcomes and increased mortality in patients with COVID-19 [12]. Pneumonia was common in COVID-19 affecting up to $90 \%$ of cases in China [13]. In general, the mortality risk due to pneumonia in people with dementia has been reported to be twice that in people without dementia [14] (Fig. 1).

\section{COVID-19 and Dementia—the Consequences}

Health care systems are adapting to absorb the pressure of treating the COVID-19 affected cases with creation of more acute and intensive care unit (ICU) beds. This may divert the focus of care from chronic conditions such as dementia to handle the acute situation. As a result, the specific care needs of older people with dementia may be compromised or less prioritised. Regular elective monitoring of older people with dementia will be affected as well as delay in diagnosing new cases may occur. Non-pharmacological interventions that depend on physical and social contact such as pet therapy or group exercise may result in cognitive, affective and physical deterioration. Also, upon recovery from this pandemic, the demand on care to compensate for this backlog will be overwhelming for the health and social care to cope with. Many cases may have been developing complications and need more medical attention, and many families will need more social support to look after their progressively disabled patients. On the other hand, carers themselves may become unavailable due to increased demand, their own illness or they are required to self-isolate due to exposure to positive cases. Other serious consequences are in the care home settings. On one hand, the prevalence of dementia is high, especially those with advanced disease and behavioural changes such as 
Fig. 1 Dementia and COVID-19: the link, the risk and the increased vulnerability to infection and worse outcomes

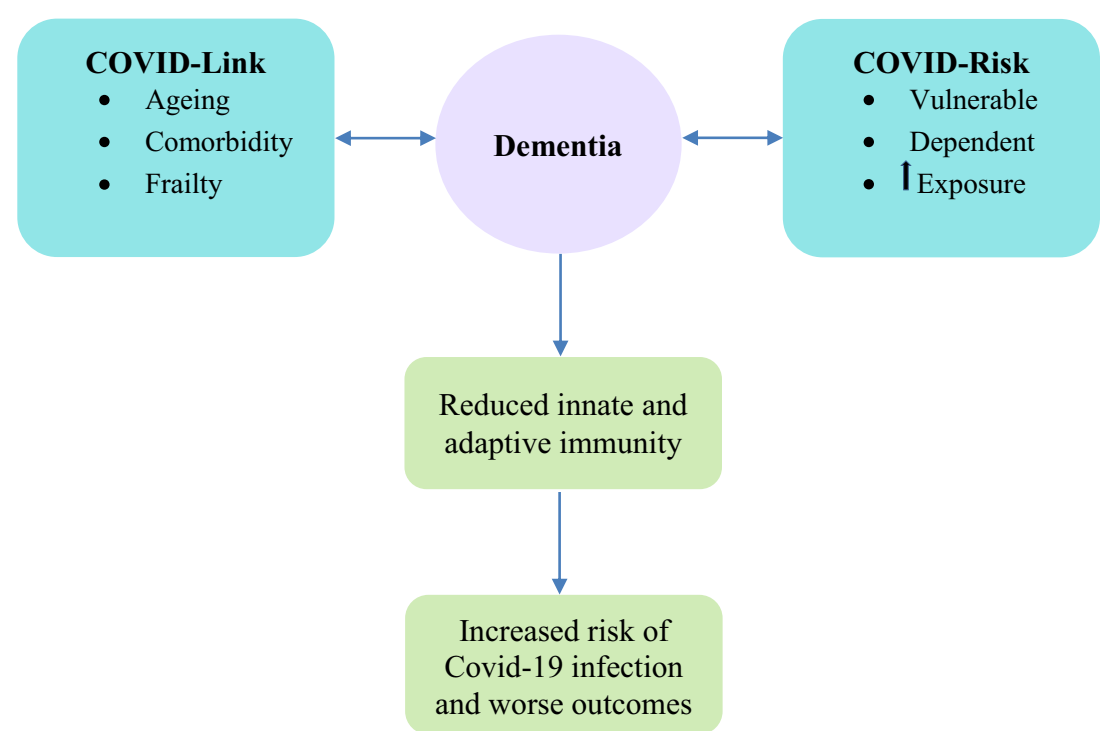

increased agitation and wondering, and on the other hand is the crowdedness and increased risk of infecting other residents increasing hospitalisation burden and mortality risk. This has been demonstrated in a US long-term care facility report of 101 residents with COVID-19; the median age was 83 years (range, 51 to 100 ) where both hospitalisation $(54.5 \%)$ and case fatality rates (33.7\%) were high [15]. The feasibility of clinical studies may also be abruptly put on hold, which may have long-term negative consequences in the field of cognitive dysfunction research.

\section{Management}

Older people living with dementia and have COVID-19 infection will need special care during their hospital admission due to their complex needs (Table 1).

\section{Acute Care}

Patients with dementia may be hospitalised due to deterioration of dementia itself, COVID-19 infection, other acute medical illness or not able to cope at home due to deterioration in their function or due to a break in their supportive service. Hospital-acquired COVID-19 infection is relatively high due to the vulnerability of older people with dementia, high number and turnover of health care staff and high number of patients. Some patients will also be asymptomatic carriers, and given the limited spaces available, that is not suitable for adequate social distancing, risk of cross infection will increase. In addition, limited supply of the personal protective equipment may also be an issue. Some people with serious COVID19 infection may need ICU admission which may lead to increased stress, behavioural changes and delirium [16]. Delirium caused by COVID-19 could complicate the symptoms of dementia, increase patients suffering and complicate the care need for this population [17]. Therefore,

Table 1 Summary of care for older people with dementia and COVID19

Acute care

- Screen for delirium

- Avoid precipitating factors for delirium

- Reduce impact of isolation

- Early mobilisation

- Early escalation plans

Transitional care

- Well prepared to meet mental health needs

- Supportive environment for patients and carers

- Collaborative approach between primary and secondary care

- Early engagement of social services

- Vigilant observation for COVID-19 symptoms recurrence

Long-term care

- Multidisciplinary holistic assessment

- Patients and carers education about COVID-19 prevention especially

in care homes

- Long-term psychosocial support

- Screening for anxiety and depression

- Introduction of telehealth

Palliative care

- Early screening for frailty

- Palliation considered from outset

- Holistic and compassionate approach

- Early family communications

- Early palliative team involvement

Ethical issues

- DNAR decisions clearly discussed with patients and/or carers

- Early discussions about ceiling of care

- Escalation plans based on biological rather than chronological age

- Participation in research should be based only on patient' interest

- Advanced directives discussed prior to hospital discharge 
clinicians should be aware of the high risk of dementia patients for COVID-19 infection and also for the atypical presentation with delirium if they get infected. A rapid screening tool for delirium is the 4 AT which may take only 2 min to do. The 4AT is a well-validated tool that shows good diagnostic accuracy with a pooled sensitivity of 0.88 (95\% CI 0.80 to $0.93)$ and pooled specificity of 0.88 (95\% CI 0.82 to 0.92$)$ in older ( $\geq 65$ years) people in various care settings that include emergency department, medical, stroke and surgical wards [18]. Precipitating factors for delirium such as sleep deprivation, pain, constipation and urinary retention should be avoided or promptly treated as possible.

\section{Transitional Care}

Transitional care is a model of care aiming at supporting patients and their carers after their hospital discharge to maintain their health and quality of life while awaiting to be medically and functionally ready for home. Although transitional care is usually designed to look after the physical needs of patients with dementia and COVID-19, it should also be prepared to meet their mental health requirements such as cognitive decline and behavioural change demands. This is to address the complex needs of older people with dementia affected by COVID-19 throughout their prolonged recovery period [19]. Some medically fit patients for discharge are not able to leave hospital due to unavailability of their original carers due to illness, and a new formal care will need to be re-organised or informal care be provided by family members in the interim period. Also, individuals with dementia who are normally residents in care homes and admitted to hospital may not be able to be discharged back to their residency due to the increased risk of infection associated with living in large groups. As a result, respite care with a family support may be required. Mutual trust between patients and staff, patients' involvement in their own decision-making, well-coordinated collaboration between health care and social services that includes symptom control and continuity of care are the fundamentals of successful transitional care [20]. This approach will effectively address the holistic needs of the vulnerable older people with dementia and COVID-19 and cares for this population as they transition back from the hospital to the community. Care should include a comprehensive assessment of recurrence of COVID-19 symptoms and also be aware that carers may develop COVID-19 themselves as well as their mental health responses to the crisis should be closely monitored.

\section{Long-Term Care}

Multidisciplinary teams approach to address a holistic care for patients with dementia, and their families and carers may minimise the negative impact of COVID-19 on this population. In addition to physical protection from virus infection, mental health and psychosocial support should be considered. For example, collaboration between mental health professionals, social workers, nursing home administrators and volunteers may help deliver a holistic care. Educating patients, if appropriate, and their carers for relaxation and meditation exercises may help reduce stress. Telehealth may be utilised for delivering behavioural management support or online consultations [21]. Effective and culturally sensitive strategies should be considered in the development of the digital forms of communication such as engaging people from diverse racial and ethnic backgrounds to ensure that language competency and cultural values are addressed into care plans [22]. Long-term monitoring is essential as stress and trauma can accelerate cognitive decline in addition to the impact of old age, illness, depression, trauma and dementia that may increase the risk for suicide [23, 24]. Long-term mental health effects on the carers such as post-traumatic stress disorder and depression should be monitored. During COVID-19 confinement, a telephonebased survey was administered in Spain to 93 participants with mild cognitive impairment or mild dementia in the TVassisted clinical trial and showed that television was a preferred technological device to access COVID-19 information and perform recreational activity and memory exercises. The results suggest that television-based telehealth support using TV-assisted demonstrated potential for cognitive stimulation [25].

\section{Palliative Care}

Many older people with dementia affected by COVID-19 are frail and approaching their terminal phase of life expectancy. Therefore, palliation should be considered from the outset. The relief of suffering, through a holistic and compassionate approach, is an essential component of care for patients with critical or life-threatening illness. Palliative care is not only caring for dying patients but it is concerned with management of distressing symptoms, planning ahead, communicating with patients and their families, grief and bereavement.

Referral to palliative care team should be considered early on in patients with COVID-19 as symptoms can escalate very rapidly. In a Chinese study, the median time from onset of symptom to breathlessness was 5 days, and to acute respiratory distress syndrome (ARDS) was 8 days [26]. Similarly, in an Italian study, the median time from the first symptom to hospitalisation was 5 days, and to death was 9 days [27]. The most common symptoms for patients with COVID-19 referred for palliation were breathlessness, agitation, drowsiness and delirium [28]. Opioids are the main agents used for the relief of severe breathlessness [29]. Oxygen therapy is another option for the relief of breathlessness in patients with severe hypoxia. There is no evidence to support the use of oxygen for breathlessness in the absence of hypoxia [30]. Agitation and anxiety are common symptoms among people with severe 
Covid-19 and can be managed with administration of benzodiazepines alone or in combination with opioids [31]. If delirium is contributing to agitation or anxiety, an antipsychotic such as haloperidol may be used instead of, or in addition to benzodiazepines. Pre-emptive medications for symptom management should be available when the initial care plan is put in place, alongside the acute medical management plan, in the event of acute or sudden deterioration.

\section{Ethical Issues}

Decisions around "do not attempt resuscitation (DNAR)" should be made early on admission to hospital after discussion with patients, if appropriate, and/or their carers. Issues such as accessibility to ICU beds for older people with dementia or the decision to take them off the ventilator, if they are not responding to treatment, in view of the limited bed availability are controversial ethical issues that need to be addressed early on after admission. Overall prognosis and best chance of survival based on biological age rather than chronological age is one way of selecting patients to a higher level of care and continuation of intensive therapy. The National Institute of Clinical Excellence (NICE) has suggested using the CFS which measures overall function independent of age, as a tool of predicting prognosis and selection of patients for ICU admission. This objective tool may also help protect psychological well-being of the health care professionals making such difficult decisions. Another ethical issue is the participation of older people with dementia in clinical trials. For example, research activities in which participants may be exposed to potential infection, through face-to-face contact with research staff, without clear direct benefit for them should be suspended unless it can be conducted safely by a remote communication or another way that minimises the risk of infection. This should be explicitly discussed with the patients and their carers explaining clearly risks and benefits of taking part in the clinical study. Also, decisions regarding advanced directives are needed prior to hospital discharge and should be clearly documented in medical records after discussion with patients and their carers.

\section{Future Perspectives}

The world is facing a global epidemic of both ageing and dementia. By the year 2050, the total number of people aged $\geq 60$ years are expected to reach 2 billion from 900 million in 2015. The fastest growing group is those above the age of 80 years who are expected to reach 434 million in 2050 [32]. The global ageing, which started initially in high income countries, is now affecting all countries, and almost $80 \%$ of the older people will be residing in the low and middle-income countries by the year 2050 [32]. Dementia is also emerging as a global pandemic in older people with more than 50 million people currently living with dementia and the incidence increases by one new case every 3 s [33]. Dementia, a disease of old age, is likely to be associated with a variety of comorbidities, especially frailty, which makes this group of patients vulnerable to infection. In addition, they tend to live in a crowded environment such as care homes and needing continuous contact with carers which may further increase their risk of infection. Dementia has been shown to be associated with a significant increased risk of mortality in patients with COVID-19 infection [34]. Research is ongoing to develop effective anti-COVID-19 agents as well as protective vaccination. Older people with comorbid dementia and frailty have compromised immunity that needs to be considered in current vaccination research to guarantee good immune response and efficacy in this vulnerable population. With global ageing, the number and proportion of older people with dementia will continue to increase, especially in the low-and-middle income countries which have limited resources to face any further pandemic with an increased risk of uncontrollable global spread. Therefore, WHO and world-wide policy makers must take this into account in their future planning for health care. It is a global task to reduce frailty and comorbidity and to promote healthy ageing through adoption of a healthy life style and a widespread access to preventative health care across the world especially in the deprived societies. New ways of remote communication with people living with dementia still require future investigations. Additionally, care home populations, which tend to have high prevalence of dementia, are likely to continue to increase, and these crowded settings are a risk factor for the spread of infection; therefore, review of current policies and novel regulations are required.

\section{Conclusion}

Globally, people living with dementia are at an increased risk of COVID-19 infection and worse outcomes. Patients with dementia admitted to hospital with COVID-19 tend to present atypically with neurological symptoms such as delirium. Due to their complex needs, older people with comorbid dementia and COVID-19 will require a multidisciplinary approach and continuous support during their acute illness, transitional period and long-term care. The introduction of telehealth programmes that are suitable for old age with poor cognitive function and cover diverse cultural backgrounds are urgently required for future support of this vulnerable group of patients. 
Authors' Contributions Both DE and AHA have contributed equally to literature search, writing and editing of the manuscript.

Data Availability Statement No data available as it is a review article.

\section{Compliance with Ethical Standards}

Conflict of Interest The authors declare that they have no conflict of interest.

Ethical Approval N/A.

Informed Consent N/A

\section{References}

Wu Z, McGoogan J. Characteristics of and important lessons from the coronavirus disease 2019 (COVID-19) outbreak in China. JAMA. 2020;323:1239. https://jamanetwork.com/journals/jama/fullarticle/ 2762130. Accessed 01/10/2020

2. COVID-19: epidemiology, virology and clinical features. GOV.UK. 2020. https://www.gov.uk/government/publications/ wuhan-novel-coronavirus-background-information/wuhan-novelcoronavirus-epidemiology-virology-and-clinical-features. Accessed 01/10/2020

3. Hui DS, Wong PC, Wang C. SARS: clinical features and diagnosis. Respirol. 2003;8(Suppl):S20-4.

4. Dementia-World Health Organisation. Accessed $14^{\text {th }}$ Sept 2020.

5. Emmerton D, Khan S, Conway J, Mosby D, Abdelhafiz AH. Ageing, comorbidity and frailty-synergistic risk factors for covid19 adverse outcomes. OBM Geriatrics. 2020;4. https://doi.org/10. 21926/obm.geriatr.2003127.

6. Williamson E, Walker AJ, Bhaskaran K, et al. OpenSAFELY: factors associated with COVID-19-related hospital death in the linked electronic health records of 17 million adult NHS patients. medRxiv. https://doi.org/10.1101/2020.05.06.20092999.

7. Guan WJ, Liang WH, Zhao Y, Liang HR, Chen ZS, Li YM, et al. Comorbidity and its impact on 1590 patients with Covid-19 in China: a nationwide analysis. Eur Respir J. 2020;55:2000547. https://doi.org/10.1183/13993003.00547-2020.

8. Kulmala J, Nykänen I, Mänty M, Hartikainen S. Association between frailty and dementia: a population-based study. Gerontology. 2014;60:16-21.

9. Buchman AS, Yu L, Wilson RS, et al. Association of brain pathology with the progression of frailty in older adults. Neurology. 2013;80:1-7.

10. Hewitt J, Carter B, Vilches-Moraga A, Quinn TJ, Braude P, Verduri A, et al. The effect of frailty on survival in patients with COVID-19 (COPE): a multicentre, European, observational cohort study. Lancet. 2020;5:e444-51. https://doi.org/10.1016/S2468-2667(20) 30146-8.

11. Bauer K, Schwarzkopf L, Graessel E, et al. A claims data-based comparison of comorbidity in individuals with and without dementia. BMC Geriatr. 2014;14:1-13.

12. Zhou F, Yu T, Du R, et al. Clinical course and risk factors for mortality of adult inpatients with COVID-19 in Wuhan, China: a retrospective cohort study. Lancet. 2020;395:1054-62.

13. Guan W, Ni Z, Hu Y, Liang WH, Ou CQ, He JX, et al. Clinical characteristics of coronavirus disease 2019 in China. N Engl J Med. 2020;382:1708-20.
14. Foley NC, Affoo RH, Martin RE. A systematic review and metaanalysis examining pneumonia-associated mortality in dementia. Dement Geriatr Cogn Disord. 2015;39:52-67.

15. McMichael TM, Currie DW, Clark S, Pogosjans S, Kay M, Schwartz NG, et al. Epidemiology of Covid-19 in a long-term care facility in king county. Washington NEJM. 2020;382:2005-11. https://doi.org/10.1056/NEJMoa2005412.

16. Kales HC, Lyketsos CG, Miller EM, Ballard C. Management of behavioral and psychological symptoms in people with Alzheimer's disease: an international Delphi consensus. Int Psychogeriatr. 2019;31:83-90.

17. Marcantonio ER. Delirium in hospitalized older adults. N Engl J Med. 2017;377:1456-66.

18. Tieges Z, Maclullich AMJ, Anand A, et al. Diagnostic Accuracy of the 4AT for delirium detection: systematic review and meta-analysis. medRxiv. 2020. https://doi.org/10.1101/2020.06.11.20128280.

19. Naylor MD, Hirschman KB, Hanlon AL, Bowles KH, Bradway C, McCauley KM, et al. Comparison of evidence-based interventions on outcomes of hospitalized, cognitively impaired older adults. J Comp Eff Res. 2014;3:245-57.

20. Emmerton D, Abdelhafiz A. Delirium in older people with COVID19: clinical scenario and literature review. SN Compr Clin Med. 2020;2:1790-7.

21. Boots LMM, de Vugt ME, van Knippenberg RJM, Kempen GIJM, Verhey FRJ. A systematic review of internet-based supportive interventions for caregivers of patients with dementia. Int $\mathrm{J}$ Geriatr Psychiatry. 2014;29:331-44.

22. Barclay G, Sabina A, Graham G. Population health and technology: placing people first. Am J Public Health. 2014;104:2246-7.

23. Tsolaki M, Papaliagkas V, Kounti F, Messini C, Boziki M, Anogianakis G, et al. Severely stressful events and dementia: a study of an elderly Greek demented population. Psychiatry Res. 2010;176:51-4.

24. Alphs L, Brashear HR, Chappell P, Conwell Y, Dubrava S, Khin NA, et al. Considerations for the assessment of suicidal ideation and behavior in older adults with cognitive decline and dementia. Alzheimers Dement Transl Res Clin Interv. 2016;2:48-59.

25. Goodman-Casanova JM, Dura-Perez E, Guzman-Parra J, CuestaVargas A, Mayoral-Cleries F. Telehealth home support during COVID-19 confinement for community-dwelling older adults with mild cognitive impairment or mild dementia: survey study. J Med Internet Res. 2020;22:e19434.

26. Wang D, Hu B, Hu C, Zhu F, Liu X, Zhang J, et al. Clinical characteristics of 138 hospitalized patients with 2019 novel coronavirus-infected pneumonia in Wuhan, China. JAMA. 2020;323:1061-9.

27. COVID-19 Surveillance Group. Characteristics of COVID-19 patients dying in Italy. Report based on available data on March 26th, 2020. https://www.epicentro.iss.it/coronavirus/bollettino/ReportCOVID-2019 26 marzo eng.pdf.

28. Lovell N, Maddocks M, Etkind SN, Taylor K, Carey I, Vora V, et al. Characteristics, symptom management and outcomes of 101 patients with COVID-19 referred for hospital palliative care. J Pain Symptom Manag. 2020;60:e77-81.

29. Ekström M, Bajwah S, Bland JM, Currow DC, Hussain J, Johnson MJ. One evidence base; three stories: do opioids relieve chronic breathlessness? Thorax. 2018;73:88-90.

30. Allsop M, Ziegler L, Fu Y, et al. Oxford COVID-19 Evidence Service Team. Is oxygen an effective treatment option to alleviate the symptoms of breathlessness for patients dying with COVID-19 and what are the potential harms? CEBM, 2020. https:/www.cebm. net/covid-19/is-oxygen-an-effective-treatment-option-to-alleviatethe-symptoms-of-breathlessness-for-patients-dying-with-covid-19and-what-are-the-potential-harms. Accessed 15/10/20202

31. Navigante AH, Cerchietti LC, Castro MA, et al. Midazolam as adjunct therapy to morphine in the alleviation of severe dyspnea 
perception in patients with advanced cancer. J Pain Symptom Manag. 2006;31:38-47.

32. Ageing and health. World Health Organisation. Accessed October 2020.

33. Alzheimer's Disease International World Alzheimer's report 2019: attitudes to dementia. https://www.alz.co.uk/research/ WorldAlzheimerReport2019.pdf. Accessed 15/10/2020

34. Bianchetti A, Rozzini R, Guerini F, Boffelli S, Ranieri P, Minelli G, et al. Clinical presentation of COVID-19 in dementia patients. J Nutr Health Aging. 2020;24:560-2.
Key points

- People living with dementia are at risk of COVID-19 infection and worse outcomes.

- Continuous multidisciplinary support for people with comorbid dementia and COVID-19 during their acute illness, transitional period and long-term care is required.

- Telehealth may be a promising safe way to look after this vulnerable group of patients in the future.

Publisher's Note Springer Nature remains neutral with regard to jurisdictional claims in published maps and institutional affiliations. 